\title{
Low DLG2 gene expression, a link between 11q-deleted and MYCN-amplified neuroblastoma, causes forced cell cycle progression, and predicts poor patient survival
}

\author{
Simon Keane', Sophie Améen ${ }^{1}$, Angelica Lindlöf ${ }^{2}$ and Katarina Ejeskärr ${ }^{1 *}$
}

\begin{abstract}
Background: Neuroblastoma (NB) is a childhood neural crest tumor. There are two groups of aggressive NBs, one with MYCN amplification, and another with 11q chromosomal deletion; these chromosomal aberrations are generally mutually exclusive. The DLG2 gene resides in the 11 -deleted region, thus makes it an interesting NB candidate tumor suppressor gene.

Methods: We evaluated the association of DLG2 gene expression in NB with patient outcomes, stage and MYCN status, using online microarray data combining independent NB patient data sets. Functional studies were also conducted using NB cell models and the fruit fly.

Results: Using the array data we concluded that higher DLG2 expression was positively correlated to patient survival. We could also see that expression of DLG2 was inversely correlated with MYCN status and tumor stage. Cell proliferation was lowered in both 11q-normal and 11q-deleted NB cells after DLG2 over expression, and increased in 11q-normal NB cells after DLG2 silencing. Higher level of DLG2 increased the percentage of cells in the G2/M phase and decreased the percentage of cells in the G1 phase. We detected increased protein levels of Cyclin $A$ and Cyclin B in fruit fly models either over expressing dMyc or with RNAi-silenced $d m D L G$, indicating that both events resulted in enhanced cell cycling. Induced MYCN expression in NB cells lowered DLG2 gene expression, which was confirmed in the fly; when dMyc was over expressed, the dmDLG protein level was lowered, indicating a link between Myc over expression and low dmDLG level.

Conclusion: We conclude that low DLG2 expression level forces cell cycle progression, and that it predicts poor NB patient survival. The low DLG2 expression level could be caused by either MYCN-amplification or 11q-deletion.
\end{abstract}

Keywords: Neuroblastoma, 11q, MAGUK, DLG2, MYCN

\footnotetext{
* Correspondence: katarina.ejeskar@his.se

'Translational Medicine, School of Health Sciences, University of Skövde, PO Box 408, SE-54128 Skövde, Sweden

Full list of author information is available at the end of the article
}

C C The Author(s). 2020 Open Access This article is licensed under a Creative Commons Attribution 4.0 International License, which permits use, sharing, adaptation, distribution and reproduction in any medium or format, as long as you give appropriate credit to the original author(s) and the source, provide a link to the Creative Commons licence, and indicate if changes were made. The images or other third party material in this article are included in the article's Creative Commons licence, unless indicated otherwise in a credit line to the material. If material is not included in the article's Creative Commons licence and your intended use is not permitted by statutory regulation or exceeds the permitted use, you will need to obtain permission directly from the copyright holder. To view a copy of this licence, visit http://creativecommons.org/licenses/by/4.0/ The Creative Commons Public Domain Dedication waiver (http://creativecommons.org/publicdomain/zero/1.0/) applies to the data made available in this article, unless otherwise stated in a credit line to the data. 


\section{Background}

Neuroblastoma (NB) is a tumor arising from the transient embryonic neural crest, later presenting in the sympathetic division of the autonomous nervous system [1]. It is one of the most common forms of extra cranial solid tumors found in children aged under two [2]. The clinical diagnosis of NB is difficult in part due to the age of the patient and the vague appearance of the symptoms [3]. NB is post surgically staged according to the International Neuroblastoma Staging System (INSS). INSS stages 1 and 2 are complete or partially resected localized tumors; stage 3 denotes the larger localized tumors that cross the midline. Stage 4 tumors have dissemination of the tumor to lymph nodes or bone marrow. Stage $4 \mathrm{~S}$ are special cases, where the patient is younger than 1 year old, having a one sided tumor with metastasis to the liver or skin but limited bone marrow involvement [4]. The survival prognosis of the $4 S$ stage patients is lower than the stage 2 tumor group but better than the stage 3 tumor group [5].

NB can also be defined by risk group, low and intermediate NB have a good prospect for treatment, whereas, the high risk tumors are more difficult to treat [6] and which results in a higher mortality. High-risk NB tumors have specific genetic characteristics and are highly aggressive. Two of the most prevalent forms of high risk NB are; amplification of the $M Y C N$ proto-oncogene [7, 8] and unbalanced 11q-deleted loss of heterozygosity ( $\mathrm{LOH}$ ) tumors $[2,9]$, which account for approximately 20 and 30\%, respectively, of all cases. Patients with $11 \mathrm{q} \mathrm{LOH}$ are always heterozygous for the deletion and generally lack $M Y C N$ amplification [10]. However, in the rare cases when patients do have both $M Y C N$ amplification and 11q LOH, the deletion point is found to be located more terminally than other $11 \mathrm{q} \mathrm{LOH}$ tumors. These two common aggressive NB forms are therefore usually considered to be mutually exclusive [11]. The mechanism of the mutual exclusivity remains to be determined, as the definitive identification of a single tumor suppressor gene within $11 q$ remains elusive. Consequently, it is accepted that the tumor suppressor gene disrupted by 11q LOH must satisfy a set of criteria based on the known clinical and genetic alterations observed, something that previous attempts at tumor suppressor characterization on 11q have also considered. The first characteristic is the inverse relation of $11 \mathrm{q}$ and $M Y C N$ amplification; with $11 \mathrm{q}$ deletion substituting the function of $M Y C N$ amplification or by $M Y C N$ amplification acting to disrupt the $11 \mathrm{q}$ tumor suppressor gene function [11]. Secondly, the tumor suppressor should maintain genome integrity and prevent the increased number of chromosomal breaks that are observed in the 11q LOH tumors [2]. Finally, there should also be a suitable two hit mechanism to account for the deletion always appearing as heterozygous only deletion $[12,13]$. To date a second hit has not been found in the proposed 11q tumor suppressor genes, including; CADM1 (11q23.3) [14], ATM (11q22.3) [15] and H2AFX (11q23.3) [11].

Since the identification of $11 \mathrm{q}$-deleted NB, debate has raged over the smallest region of overlap (SRO) of the deletion. Initially, the SRO was identified at 11q23 [16], subsequent identification showed that the deletion extended to 11q14 [10]. Now it has been suggested that there are three separate SROs on 11q; the first, an amplification from 11q13.2 to 11q13.4, the second, a deletion spanning from $11 \mathrm{q} 14.1$ to $11 \mathrm{q} 22.2$ and finally a deletion spanning 11q23.1 to $11 \mathrm{q} 23.3$ for the rare tumors with both $11 \mathrm{q}$ deletion and $M Y C N$ amplification [17]. Located within the 11q14.1 SRO, and always deleted in the $11 q$-deleted NBs without $M Y C N$ amplification, is one of the Discs Large homolog (DLG) family members, DLG2. The DLG family has important functions governing polarity, cellular structure and growth behavior [18-20]. These functions are thought to be achieved by protein trafficking to the cellular surface of epithelial cells as well as the organization and stabilization of supramolecular adhesion and signaling complexes through heterodimeric formation [21]. Orthologous to the human DLG2 gene is the Drosophila melanogaster DLG gene (dmDLG). Drosophila melanogaster only contain a single orthologue to the human DLG family. Loss or mutation of $d m D L G$ is known to result in spontaneous neoplasms [22]. $d m D L G$ was identified early as a tumor suppressor gene along with complex partners scribble (Scrib) and lethal giant larvae (LGL) [23]. Knockdown of $d m D L G$ has been implicated in early and abnormal exit from the cell cycle and can result in binucleate cells [24]. $d m D L G$ has also been shown to be important in Drosophila spindle alignment in asymmetric neuroblast division [25]. Recently, abnormally low DLG2 expression in the human cancers osteosarcoma [26] and ovarian cancer [27] has been identified. GWAS studies have also shown that an intronic SNP (rs790356) within DLG2 correlates to nephroblastoma, a common childhood renal tumor [28]. Therefore, DLG2 is a good candidate to be the $11 \mathrm{q}$ tumor suppressor gene in NB.

In this study we have considered $D L G 2$ to be a functional and positional tumor suppressor gene in 11qdeleted NB. We have investigated this using clinical pathology data and gene expression in both primary NBs and in NB cell lines. We have confirmed this data by $D L G 2$ over expression and silencing experiments in $\mathrm{NB}$ cell lines and in the fruit fly.

\section{Methods}

\section{Gene expression analysis}

Data for analyses and comparison of DLG2 expression between the different $\mathrm{NB}$ patient subgroups was imported from the R2 platform (http://r2.amc.nl). The 
independent NB primary datasets; 1): SEQC GSE49710 (microarray), 2): Versteeg GSE16476 (microarray), 3): Maris GSE3960 (microarray) and 4): Westermann GSE73517 (microarray). Neuroblastoma methylation datasets; 1): Westermann GSE73515 (Methylation array) and 2): Fisher GSE120650 (Methylation array). The neuroblastoma cell datasets; Maris; 1): GSE89413 (Cell Line, RNA-Seq), 2): Versteeg GSE28019 (Cell Line, microarray), 3), Versteeg GSE16478 (Cell Line, microarray), and human embryogenesis dataset 1): Yi GSE15744 (microarray). The microarray data was downloaded as the centered $\log 2$ fold change. Methylation data was downloaded as raw values.

\section{Cell lines and tissue culture}

Human NB cell line SKNAS and NB69 were obtained from ATCC Cell Line Collection. The cell lines were maintained in RPMI 1640 supplemented with 10\% FBS, 1\% L-Glutamine, 1\% HEPES solution and 1\% sodium pyruvate. Cells were maintained at $37{ }^{\circ} \mathrm{C}$ with $5 \% \mathrm{CO}_{2}$.

\section{Plasmids, siRNAs and transfections}

DLG2 (NM_001364) expression plasmids on a backbone of pCMV6-AC-GFP (catalogue \# PS100010) vector were purchased from Origene Technologies. siRNA targeting DLG2 (s4122) or Silencer ${ }^{\text {TM }}$ Select Negative control No. 1 siRNA (4390843) was purchased from Ambion (Thermo Fischer Scientific). SKNAS and NB69 cells were grown to $80 \%$ confluence and subsequently transfected with; $D L G 2$ plasmid, empty vector "mock" (pCMV6-AC-GFP), si-DLG2 or scrambled control "mock". 100 ng of plasmid DNA or 10 pmol siRNA was complexed with $0.3 \mu \mathrm{l}$ of Lipofectamine 2000 according to the Lipofectamine 2000 reagent forward transfection protocol (Invitrogen; Thermo Fisher Scientific).

\section{Cell viability, proliferation and cell cycle analysis}

$100 \mu \mathrm{l}$ cell suspension of SKNAS and NB69 $\left(1 \times 10^{4}\right.$ cells/well) was seeded in 96-well culture plates (Corning Incorporated). After culturing to $80 \%$ confluence the supernatant was removed and transfection media was added to the cells. $48 \mathrm{~h}$ post transfection, cells were counted using a $60 \mu \mathrm{m}$ sensor for the Scepter handheld cell counter (Millipore) as per the manufactures instructions [29]. Cell proliferation was measured using the MTS/MPS Cell Titer $96^{\circ}$ One solution Reagent (Promega) and detecting the color variation (FLUOstar Omega, BMG Labtech) as per the manufacturer's recommendations. The absorbance values were normalized to the mock transfection and expressed as a percentage. All experiments were repeated three times.

Cell cycle analysis was performed using the Cell-clock cell cycle assay (Biocolor). Images were subsequently analyzed using Image $\mathrm{J}$ image analysis as per the manufacturer's instructions. The data presented is the average of three biological replicates. Each experiment series was repeated in triplicate.

\section{Fly strains and crosses}

Commercially available control white (w1118) flies, UAS-dMYC and UAS-RNAi-dlg1 flies were crossed with da-GAL4 driver strain to ubiquitously force or silence gene expression, all strains were obtained from the Bloomington stock center (Bloomington). Twenty female da-GAL4 flies were crossed with 10 male UAS-transgenic flies or control flies and the progeny incubated at $25^{\circ} \mathrm{C}$ on standard fly media. Five of the progeny were harvested after $72 \mathrm{~h}$ during the third instar larvae phase. Each cross was controlled using the inverse cross using 20 UAS-transgenic female flies crossed with $10 \mathrm{da}-$ GAL4 male flies.

\section{Protein analysis by Western blot}

Protein was extracted from the transfected cells in 96 well plates $\left(1 \times 10^{4}\right.$ cells/well), by aspirating the media and incubating on ice for $5 \mathrm{~min}$ then adding ice cold RIPA buffer (Thermo-fisher Scientific, 89900). For fly protein extractions 5 larvae were homogenized in RIPA buffer, followed by centrifugation. Western blot analysis was performed using a Mini-PROTEAN ${ }^{\bullet}$ TGX $^{\mathrm{TM}} 8-20 \%$ gradient gel (Bio-Rad), protein was blotted onto LFPVDF membrane (8 min, $25 \mathrm{~V}$ and $2.5 \mathrm{~A}$ ) using a TransBlot $^{\ominus}$ Turbo $^{\text {ma }}$ Transfer System (Bio-Rad). Blots were subsequently blocked for $1 \mathrm{~h}$ in $5 \%$ milk in TBST buffer $(0.1 \%$ Tween-20 and $150 \mathrm{mM} \mathrm{NaCl}$ in $10 \mathrm{mM}$ TrisHCL, pH 7.4) as per the manufacturer's recommendations. Blots were probed overnight at $4{ }^{\circ} \mathrm{C}$ with antibodies diluted in PBST (0.1\% Tween-20 in PBS). Primary antibodies; dmDLG (4F3, anti-discs large, Goodman, C.), Cyclin A (A12, Lehner, C.F.), Cyclin B (F2F4, O'Farrell, P.H.) and $\alpha$-tubulin (12G10, Frankel, J. / Nelsen, E.M.) were obtained from the Developmental Studies Hybridoma Bank, created by the NICHD of the $\mathrm{NIH}$ and maintained at The University of Iowa, Department of Biology, Iowa City, IA 52242. All primary antibodies were diluted to $0.5 \mu \mathrm{g} / \mathrm{ml}$ in PBST $0.1 \%$. The secondary antibody used for detection was Starbright goat anti-mouse 1:5000 (12004159, Bio-Rad). All wash stages were $3 \times 10$ minutes in TBST $0.1 \%$. Secondary antibodies were incubated for $1 \mathrm{~h}$ at room temperature. Image detection was performed on ChemiDoc MP (Bio-Rad).

\section{Quantitative PCR analysis}

RNA from NB cell lines was extracted with RNeasy Kit ${ }^{\circ}$ (Qiagen) according to manufacturer's protocol. RNA was quantified by NanoDrop (NanoDrop Technologies) and $2 \mu \mathrm{g}$ of RNA was reverse transcribed into double 
stranded cDNA on a T-professional Basic Gradient thermal cycler (Biometra) using the High Capacity cDNA Reverse Transcription kit (Applied Biosystems). cDNA corresponding to $20 \mathrm{ng}$ of RNA was used for each qPCR reaction. qPCR was performed on a Pikoreal qPCR System (Thermo Fischer Scientific) in triplicate for TaqMan target transcripts; DLG2 (Hs00265843_m1) and dmDLG (Dm01799281_g1) using TaqMan ${ }^{\text {тм }}$ Gene Expression Master Mix ( Applied Biosystems). Quantitative gene expression data were normalized to the expression levels of the human reference genes GAPDH ( $\mathrm{Hs} 02758991$ $\mathrm{m} 1$ ), GUSB (Hs99999904_m1) and fly reference gene Rpl32 (Dm02151827_g1).

\section{Gene set enrichment analysis}

To identify the pathways to which DLG2 expression is correlated with, the previously described independent datasets; 1, 2 and 3 were selected from the R2 platform (http://r2.amc.nl). A gene expression list was derived based on the correlation to DLG2 expression, normalized to z-score. To further investigate enriched pathways, the non-NB developmental dataset 10 was selected for additional comparison. The top 10 enriched KEGG pathways were subsequently shown for each dataset using the R2 platform. Data was corrected for multiple comparisons using the false discovery rate (FDR). Concordance between the datasets was shown using Venn diagrams produced with the geneVenn tool (genevenn. sourceforge.net).

\section{Statistical analysis}

All data is presented as Tukeys box and whisker plots showing IQR, line at the median, + at the mean with whiskers \pm 1.5 -fold of interquartile range from at least 3 independent experiments. For all multi-group analyses, differences were determined by one way ANOVA test followed by Holm-Sidak's multiple comparison test. For comparisons between two groups a Mann-Whitney $U$ test was used. For determination of overall and event free survivals the Kaplan Meier estimator was used with differences between groups determined using a two sided log rank test. $p<0.05$, $p<0.01, \quad p<0.001$. All analyses were conducted using GraphPad Prism version 8.0.1 for Windows, (GraphPad Software, www.graphpad.com).

\section{Results}

\section{DLG2 expression is low in high INSS stage NB and correlates to survival}

We evaluated the association of DLG2 expression with INSS stage as well as patient outcomes, using the NB primary dataset 1 (GSE49710) obtained from the R2 Genomics Analysis and Visualization Platform (http://r2. amc.nl). When comparing DLG2 expression level between INSS stages, stage 4 tumors showed significantly lower $D L G 2$ expression when compared to stage 1 tumors $(\log 2 \mathrm{FC}=0.89, p<0.001)$, stage 2 tumors $(\log 2 \mathrm{FC}=0.83, p<0.001)$ and stage 3 tumors $(\log 2 \mathrm{FC}=$ $0.55, p<0.005)$, with the only exception of stage $4 \mathrm{~s}$ tumors (Fig. 1a). A similar trend was also observed in the NB primary datasets 2, 3 and 4 (Fig. S1a, b and d respectively), with the stage 4 tumors showing low DLG2 expression. There was also a significant difference in the expression of DLG2 in the high risk and low risk patients $(\log 2 \mathrm{FC}=0.80, p<0.001)$ (Fig. 1b). The same trend was also observed in NB primary dataset 4 (Fig. S1f). Overall survival outcomes (Fig. 1c) and event free survival (Fig. 1d) for the patients were determined using Kaplan-Meier analysis using NB primary dataset 1 . High expression of DLG2 was associated with increased probability of both overall and event free survival (Fig. 1c-d, $p<0.001)$.). The same trend was also observed in $\mathrm{NB}$ primary dataset 2 (Fig. S2a and S2b). The gene expression data was subsequently clustered into 4 groups using the normalized total array expression and $\mathrm{K}$ means clustering method, and the survival of each of the 4 groups was then determined using Kaplan-Meier analysis. One of the groups showed a high survival, with $98 \%$ overall survival, while the other groups showed $82 \%, 47 \%$ respectively $37 \%$ survival (Fig. 1e). The groups were subsequently named after their overall survival and DLG2 expression was determined for each group. The group with $98 \%$ survival also had the highest DLG2 expression compared to $82 \%$ survival $(\log 2 \mathrm{FC}=0.49, p<0.001)$, $47 \%$ survival $(\log 2 \mathrm{FC}=0.71, p<0.001)$ and $37 \%$ survival $(\log 2 \mathrm{FC}=0.90, p<0.001)$, there was also a significantly lower expression of DLG2 in the $37 \%$ survival group when compared to the $82 \%$ survival group (log2 $\mathrm{FC}=$ $0.41, p<0.05$ ) (Fig. 1f). The similar $\mathrm{K}$ means clustering results was also observed in NB primary dataset 2 (Fig. S2c and S2d).

\section{Gene set enrichment analysis shows DLG2 inversely correlates to cell cycle genes}

Gene lists were created for the genes correlating to DLG2 expression in NB primary datasets 1 (GSE49710), 2 (GSE16476) and 3 (GSE3960), and an embryogenesis dataset 1 (GSE15744). The NB primary dataset 4 was not analysed due to the customized probe layout, and the cell line datasets were also not included. Gene set enrichment analysis of the genes correlating to DLG2 in the selected data sets showed that the pathways; cell cycle $(p<0.0001)$, DNA replication $(p<0.0001)$, Fanconi anemia $(p<0.0001)$ and mismatch repair $(p<0.0001)$ were enriched in the three analyzed NB primary datasets (Table 1, 2 and 3). When these four identified pathways were compared to the enriched pathways in the embryogenesis dataset (Table 4), the Fanconi anemia pathway 

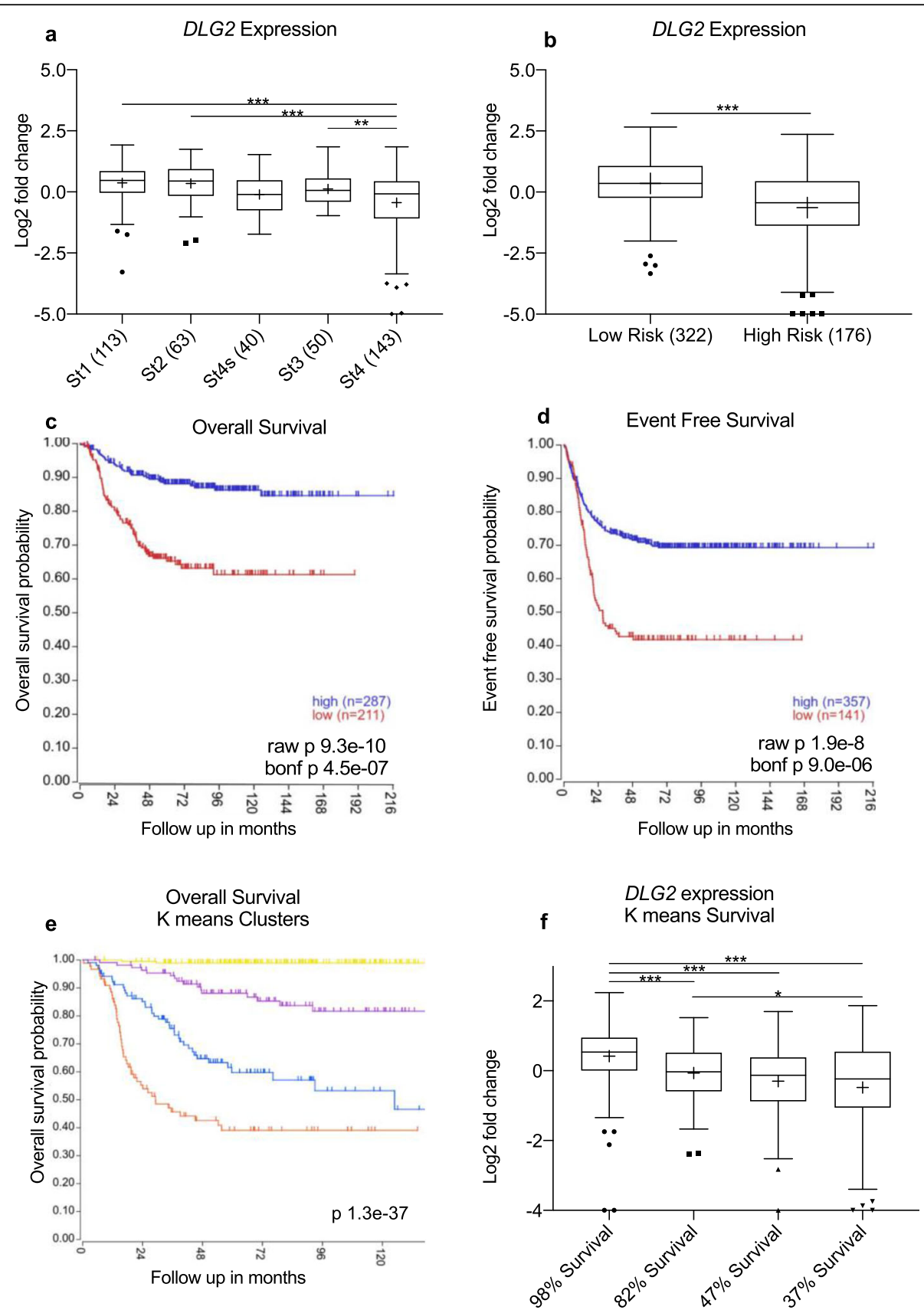

Fig. 1 DLG2 expression in NB correlates with survival and stage. DLG2 gene expression of NB primary dataset 1 (GSE49710) stratified by a INSS stage and $\mathbf{b}$ by risk category in primary NB. $\mathbf{c}, \mathbf{d}$ and $\mathbf{e}$ are Kaplan-Meier plots showing the overall survival probability, the event free survival and the overall survival of the $\mathrm{k}$ means 4 groups clustering. $\mathbf{f} D L G 2$ expression stratified by $\mathrm{K}$ means clustering. The expression data are presented as median centered log2 fold change and plotted as Tukeys box and whisker plots showing IQR, line at the median, + at the mean with whiskers \pm 1.5 -fold of interquartile range. Data outside the whiskers are shown as outliers. ${ }^{*} p<0.05,{ }^{* *} p<0.01,{ }^{* * *} p<0.001$. Kaplan-Meier plots are determined using the product limit estimator followed by Bonferroni correction

did not appear. The proportion of cell cycle genes in NB primary datasets 1,2 and 3 were $71.8 \% ; 31.4 \%$ and $47.7 \%$, respectively; $(p<0.0001)$, DNA replication genes $88.9 \%$; $52.8 \%$ and $78.1 \%$, respectively $(p<0.0001)$, mismatch repair genes $78.3 \%$; $40.9 \%$ and $70.0 \%$, respectively $(p<$ 0.0001 ) and Fanconi anemia pathway genes $69.8 \%$; $42.2 \%$ and $60.0 \%$, respectively $(p<0.0001)$ (Table 1,2 and 3 ). The embryogenesis dataset showed $41.7 \%$ cell cycle genes $(p<0.001), 61.1 \%$ DNA replication genes $(p<$ $0.0001)$ and $63.6 \%$ mismatch repair genes $(p<0.0001)$ (Table 4). The genes involved in the enriched pathways showed an overriding negative correlation to DLG2 
Table 1 GSEA of genes correlated to DLG2 expression in NB primary dataset 1 (GSE49710)

\begin{tabular}{lllll}
\hline Enriched KEGG Pathway & \# correlated genes & \# pathway genes in total & Percentage & Corrected $\boldsymbol{p}$-value \\
\hline Ribosome & 124 & 133 & $93.2 \%$ & $5.2 \mathrm{e}-25$ \\
Oxidative phosphorylation & 93 & 120 & $77.5 \%$ & $2.0 \mathrm{e}-10$ \\
Alzheimer s disease & 115 & 162 & $71.0 \%$ & $9.7 \mathrm{e}-09$ \\
Cell cycle & 89 & 124 & $71.8 \%$ & $2.1 \mathrm{e}-07$ \\
Huntington's disease & 126 & 187 & $67.4 \%$ & $2.3 \mathrm{e}-07$ \\
DNA replication & 32 & 36 & $88.9 \%$ & $1.2 \mathrm{e}-06$ \\
Sphingolipid signaling pathway & 84 & 120 & $70.0 \%$ & $2.4 \mathrm{e}-06$ \\
Spliceosome & 89 & 131 & $67.9 \%$ & $8.2 \mathrm{e}-06$ \\
Fanconi anemia pathway & 37 & 53 & $69.8 \%$ & $7.9 \mathrm{e}-03$ \\
Mismatch repair & 18 & 23 & $78.3 \%$ & $4.3 \mathrm{e}-03$
\end{tabular}

expression. Concordance between the enriched pathways for the NB primary datasets 1,2 and 3 was first determined using the intersection of these three datasets and subsequently compared to the embryogenesis dataset. The intersect of the cell cycle in the NB datasets included 25/71 genes (Fig. 2a), 13/35 genes for DNA replication (Fig. 2b), and 7/20 mismatch repair genes (Fig. $2 \mathrm{c}$ ), with the concordant genes listed in the supplementary data (Table S1). When compared to the embryogenesis dataset the intersection included 14/61 genes for cell cycle (Fig. 2d), 8/27 genes for DNA replication (Fig. 2e) and 5/17 genes for mismatch repair (Fig. 2f); these concordant genes are found in Table 5 .

\section{MYCN amplified tumors have low DLG2 expression}

Gene expression of $D L G 2$ was found to be significantly lower in MYCN amplified samples in 4 independent datasets (1-4), including, two patient cohorts and two cell line cohorts (Fig. 3a-d). The NB primary dataset 1 was used to identify the decrease in DLG2 expression in MYCN amplified samples ( $\log 2 \mathrm{FC}=0.71, p<0.0001$ ) (Fig. 3a), with NB primary dataset 2 used to confirm the result $(\log 2 \mathrm{FC}=1.21, p=0.0005)$ (Fig. $3 \mathrm{~b})$. The NB cell dataset 1 (GSE89413) with 39 distinct NB cell lines (27 containing MYCN amplification) was used to confirm the lower DLG2 expression in MYCN amplified cell lines $(\log 2 \mathrm{FC}=0.65, p=0.0022)$ (Fig. 3c). In the NB cell dataset 2 (GSE28019) we controlled for variability in the tissue of origin of the cell lines by using only the cell lines derived from metastatic bone marrow, here the $M Y C N$ amplified cells also showed lower expression $(\log 2 \mathrm{FC}=$ 1.50, $p=0.049$ ) (Fig. 3d). We further noted that induction of MYCN in the NB cell dataset 3, (GSE16478), resulted in a decrease in the expression of DLG2 (Fig. 3e). A similar trend was also observed in the NB primary datasets 3 and 4 (Fig. S1c and S1e) with the MYCN amplified tumors showing low DLG2 expression.

To model the effect of MYCN amplification on DLG2 we investigated the effect of forced expression of the orthologous Drosophila melanogaster $d M Y C$ gene using a UAS-dMYC construct ubiquitously induced by daGal4 in a fruit fly model, and investigated changes in dmDLG gene expression and protein level in the larvae. Overexpression of $d M Y C$ resulted in lower gene expression of $d m D L G$ ( $\log 2 \mathrm{FC}=0.84, p=0.001$ ) (Fig. 3f). We confirmed the decrease in dmDLG also on protein level

Table 2 GSEA of genes correlated to DLG2 expression in NB primary dataset 2 (GSE16476)

\begin{tabular}{lllll}
\hline Enriched KEGG Pathway & \# correlated genes & \# pathway genes in total & Percentage & Corrected $\boldsymbol{p}$-value \\
\hline DNA replication & 19 & 36 & $52.8 \%$ & $2.1 \mathrm{e}-11$ \\
Ribosome biogenesis in eukaryotes & 27 & 64 & $42.2 \%$ & $8.4 \mathrm{e}-11$ \\
RNA transport & 44 & 139 & $31.7 \%$ & $2.1 \mathrm{e}-09$ \\
Cell cycle & 38 & 121 & $31.4 \%$ & $3.6 \mathrm{e}-08$ \\
Fanconi anemia pathway & 19 & 45 & $42.2 \%$ & $5.0 \mathrm{e}-08$ \\
Spliceosome & 37 & 119 & $31.1 \%$ & $8.0 \mathrm{e}-08$ \\
Pyrimidine metabolism & 26 & 94 & $27.7 \%$ & $1.4 \mathrm{e}-04$ \\
Homologous recombination & 11 & 29 & $37.9 \%$ & $2.1 \mathrm{e}-04$ \\
Mismatch repair & 9 & 22 & $40.9 \%$ & $45.5 \%$ \\
Non homologous end joining & 5 & 11 & & $2.8 \mathrm{e}-04$ \\
\hline
\end{tabular}


Table 3 GSEA of genes correlated to DLG2 expression in NB primary dataset 3 (GSE3960)

\begin{tabular}{lllll}
\hline KEGG Pathway & \# correlated genes & \# pathway genes in total & Percentage & Corrected $\boldsymbol{p}$-value \\
\hline Ribosome & 57 & 70 & $81.4 \%$ & $4.50 \mathrm{e}-20$ \\
DNA replication & 25 & 32 & $78.1 \%$ & $6.70 \mathrm{e}-09$ \\
Oocyte meiosis & 42 & 80 & $52.5 \%$ & $2.60 \mathrm{e}-05$ \\
Pyrimidine metabolism & 34 & 63 & $54.0 \%$ & $6.80 \mathrm{e}-05$ \\
Cell cycle & 53 & 111 & $47.7 \%$ & $1.10 \mathrm{e}-04$ \\
Mismatch repair & 14 & 20 & $70.0 \%$ & $1.50 \mathrm{e}-04$ \\
Spliceosome & 45 & 95 & $47.4 \%$ & $4.70 \mathrm{e}-04$ \\
One carbon pool by folate & 9 & 12 & $75.0 \%$ & $9.10 \mathrm{e}-04$ \\
Fanconi anemia pathway & 15 & 25 & $60.0 \%$ & $7.60 \mathrm{e}-03$ \\
Folate biosynthesis & 8 & 11 & $72.7 \%$ & $2.60 \mathrm{e}-03$ \\
\hline
\end{tabular}

(Fig. 3g), and furthermore evaluated the effect of $d M Y C$ over expression on Cyclin A (cycA) and Cyclin B (cycB). Consistent with the previous literature [30], we could determine an increase in the expression of both cycA and $\mathrm{cycB}$ (Fig. 3g).

\section{9 deletion correlates to low DLG2 expression}

Gene expression of $D L G 2$ was found to be significantly lower in 11q-deleted NB tumor data when MYCN amplification was excluded, in two independent neuroblastoma primary datasets (3 and 4). In primary NB dataset 3 (GSE3960), the 11q-deleted samples showed a significant decrease $(\log 2 \mathrm{FC}=0.85, p=0.0004)$ when compared to the $11 \mathrm{q}$ normal tumors (Fig. $4 \mathrm{a}$ ). NB primary dataset 4 (GSE73517) confirmed that 11q-deleted tumors had lower expression of DLG2 compared to the $11 \mathrm{q}$ normal tumors $(\log 2 \mathrm{FC}=0.66, p=0.034$ ) (Fig. 4b). Methylation array data showed that there was very low methylation of the DLG2 promoter region, no observable difference in the promoter region methylation pattern was observed in 11q-deleted NB compared to 11q normal in NB methylation dataset 1 (GSE73515) (Fig. 4c) and also no DLG2 promoter methylation in general in NB methylation dataset 2 (GSE120650) (Fig. 4d).

\section{DLG2 silencing or over expression changes the growth behavior of NB cells}

Over expression of DLG2 in 11q-deleted NB cells (SKNAS) resulted in slower proliferation compared to the control (Fig. 5a, $p<0.001$ ). We observed a decrease in the number of viable cells (Fig. 5b, $p<0.001$ ) and an increase in the non-viable cell fraction (Fig. 5b, $p<0.05$ ) in cells with increased DLG2 expression. DLG2 silencing in 11q-deleted SKNAS cells resulted in a slight decrease in cell proliferation (Fig. 5a, $p<0.05$ ), with no effect in viable/non-viable cell numbers (Fig. 5b). We determined that over expression of DLG2 in SKNAS resulted in a decrease of the percentage of cells in G1 phase and an increase in the number of cells in G2/M phase (Fig. 5c). There was no difference in the cell cycle state for $D L G 2$ silenced cells compared to control (Fig. 5c). This was repeated in 11q normal NB cells (NB69), where over expression of DLG2 resulted in slower proliferation compared to the control (Fig. $5 \mathrm{~d}, p<0.001$ ). There was a significant decrease in total viable cell number (Fig. 5e, $p<0.001)$ with no increase in the nonviable fraction. Knockdown of DLG2 resulted in an increase in cell proliferation compared to the mock control in 11q normal NB69 cells (Fig. 5d, $p<0.001$ ). An increase in the total

Table 4 GSEA of genes correlated to DLG2 expression in the human embryogenesis dataset (GSE15744)

\begin{tabular}{lllll}
\hline KEGG Pathway & \# correlated genes & \# pathway genes in total & Percentage & Corrected $\boldsymbol{p}$-value \\
\hline One carbon pool by folate & 13 & 17 & $76.5 \%$ & $3.3 \mathrm{e}-05$ \\
Spliceosome & 56 & 118 & $47.5 \%$ & $4.5 \mathrm{e}-05$ \\
DNA replication & 22 & 36 & $61.1 \%$ & $5.4 \mathrm{e}-05$ \\
ECM receptor interaction & 36 & 70 & $51.4 \%$ & $1.1 \mathrm{e}-04$ \\
Mismatch repair & 14 & 22 & $63.6 \%$ & $6.4 \mathrm{e}-04$ \\
Protein digestion and absorption & 33 & 67 & $49.3 \%$ & $6.9 \mathrm{e}-04$ \\
Glycolysis Gluconeogenesis & 27 & 57 & $47.4 \%$ & $4.8 \mathrm{e}-03$ \\
Salivary secretion & 25 & 52 & $48.1 \%$ & $5.0 \mathrm{e}-03$ \\
Pyruvate metabolism & 18 & 35 & $51.4 \%$ & $6.3 \mathrm{e}-03$ \\
Cell cycle & 50 & 120 & $41.7 \%$ & $6.3 \mathrm{e}-03$ \\
\hline
\end{tabular}




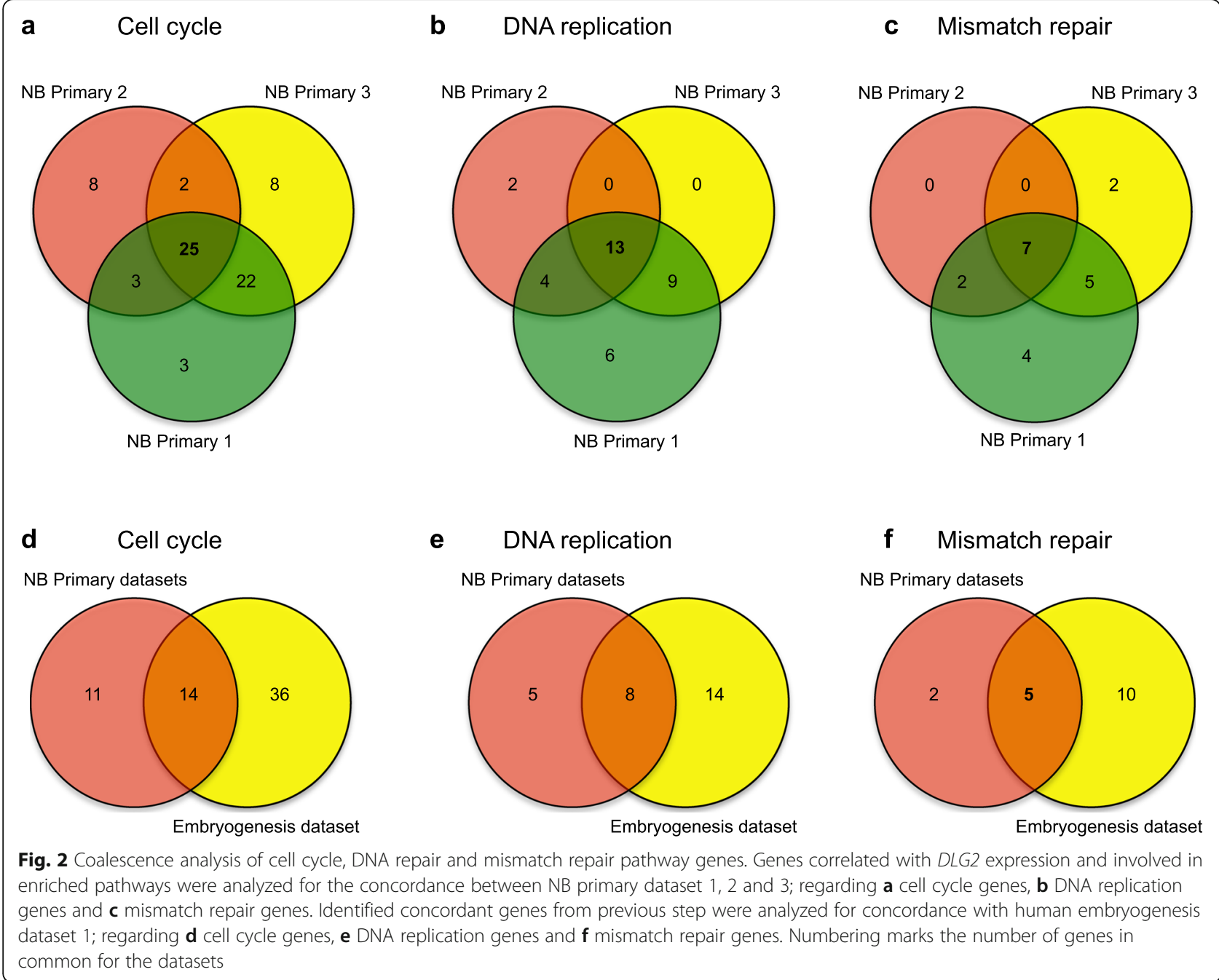

number of viable cells was also determined (Fig. 5e, $p<$ 0.001 ), with no change in the number of non-viable cells. As there was no alteration in the non-viable fraction after changing the DLG2 expression level, the changes in proliferation was not likely due to apoptosis, rather a change in cell cycle progression. Over expression of $D L G 2$ resulted in an increased fraction of cells in $\mathrm{G} 2 / \mathrm{M}$ phase (Fig. 5f, $p<0.001$ ) and the associated decrease in the fraction of cells in G1 phase (Fig. 5f, $p<0.001$ ) compared to the mock control, no difference in the number of cells in S phase was detected. Conversely, knockdown of DLG2 resulted in an increase in the number of cells in $\mathrm{G} 1$ phase and a decrease in the number of cells in $\mathrm{S}$ phase (Fig. 5f, $p<0.001$ ). Over expression and knockdown of DLG2 was confirmed using qPCR in both SKNAS and NB69 cell lines (Fig. 5g and h). To model the effect of DLG2 loss, we tested the effect of knockdown of the orthologous Drosophila dmDLG gene in a fruit fly model. RNAi-dmDLG knockdown resulted in a small decrease in $d m D L G$ RNA expression in the larvae
(Fig. 5i), the result was confirmed on protein level (Fig. 5j). To confirm if the effect on cell cycle proteins of RNAi- $d m D L G$ knockdown was similar to that of $d M Y C$ over expression (Fig. 3f) we determined the protein expression of Cyclin A and Cyclin B by Western blot. Both Cyclin A and B showed increased expression when compared to the control (Fig. 5j).

\section{Discussion}

To make the case that DLG2 is the tumor suppressor located in $11 \mathrm{q}$ we set forth a criteria based on what is currently known about the clinical and molecular features of deletion of $11 \mathrm{q}$ in NB. The first criteria we highlighted were that $11 \mathrm{q}$ deleted tumors and $M Y C N$ amplified tumors are both high-risk tumors that are difficult to treat. This difficultly to treat and resultant poor survival should be evident with altered expression when divided into INSS stage, risk category and overall survival. We could show that low DLG2 expression is associated with advanced INSS staged tumors (Fig. 1a). 
Table 5 Cell cycle, DNA replication and mismatch repair genes negatively correlated to DLG2 expression, common to NB primary datasets 1-3 (GSE49710, GSE16476 and GSE89413) and the human embryogenesis dataset (GSE15744)

\begin{tabular}{lll}
\hline Gene symbol & Gene function & Cellular process \\
\hline BUB1, BUB1B & Mitotic checkpoint serine/threonine kinase & Mitotic checkpoint \\
CCNA2 & Cyclin A2, G1/S to G2/M transition & Cell cycle regulation \\
CCNB1 & Cyclin B1, G2/M transition & Cell cycle regulation \\
CDC20 & Cell division, anaphase regulation & Chromosome separation \\
CDK4 & Cyclin dependent kinase, G1/S phase & Cell cycle regulation \\
DBF4 & E2F mediated regulation of DNA replication & DNA replication \\
ESPL1 & Sister chromatid cohesion and separation & Chromosome separation \\
EXO1 & Exonuclease 1 & DNA repair \\
LIG1 & DNA ligase, DNA replication and repair & DNA replication \\
MCM2, MCM3, MCM5, MCM7 & Initiation of genome replication & DNA replication \\
MSH6 & Mismatch recognition & DNA repair \\
POLD2 & DNA polymerase delta & DNA replication \\
PRKDC & DNA-dependent protein kinase & DNA repair \\
POLA1, POLA2 & DNA polymerase alpha & DNA replication \\
RFC3, RFC4 & Replication factor, DNA elongation & DNA replication \\
TP53 & Tumor suppressor, DNA binding & Cell cycle regulation \\
\hline
\end{tabular}

Furthermore, we could show that the overall survival and event free survival was decreased with lower expression of DLG2 (Fig. 1c and d). The second point we raised was that the prospective tumor suppressor gene (TSG) must have an interaction with $M Y C N$ amplification; either by substituting the function of $M Y C N$ amplification or by $M Y C N$ amplification acting to disrupt the 11q TSG function. We have been able to show in multiple datasets with both patient and cell models that $M Y C N$ amplification results in a decrease in the expression of DLG2. Furthermore, we could show in a fruit fly model that the overexpression of the $M Y C N$ orthologue $d M Y C$ resulted in decreased expression of Drosophila $d m D L G$ and increased expression of Cyclin A and $\mathrm{B}$ (Fig. 3g). The effect of $d M Y C$ over expression on cyclin protein level confirms previous findings [30]. After silencing $d m D L G$ expression in the fly, we could show that the outcome of the knockdown was similar to that of $d M Y C$ over expression by altering the same cyclins (Fig. 5j) and consistent with the gene set enrichment analysis (GSEA) that also verified that the genes encoding Cyclin $\mathrm{A}$ and $\mathrm{B}$ (CCNA2 and CCNB1) significantly correlated to $D L G 2$ expression in all the analyzed datasets (Table 5). We could also show that induction of $M Y C N$ in NB cells resulted in a decrease in the expression of DLG2 (Fig. 3e). Thus, supporting the case that there are common targets of MYCN amplification and 11q deletion and that MYCN acts to disrupt the 11q TSG function. It has previously been shown that MYCN has significant function controlling cell cycle and DNA repair in NB [31]. When we examined the gene sequences of DLG2 and fly $d m D L G$ we detected the MYCN/dMYC high affinity binding E-box motif CATGTG [32] appears on multiple occasions within the genes $D L G 2$ and $d m D L G$. This may provide a mechanism for $M Y C N$ to down regulate DLG2 expression, as MYCN as has previously been shown to do with regulation of FAIM2 [33], however more investigation into this mechanism is warranted. The third point that we discussed was that there must be a discernable decrease in DLG2 expression between the 11q-deleted and 11q-normal tumors, and we could indeed observe lower DLG2 expression in two NB primary datasets (Fig. 4a and b).

We highlighted that the 11q TSG must be involved in the maintenance of DNA integrity as the 11q deleted tumors often show greater chromosomal instability compared to other NB tumors [2]. The DLG2 GSEA of the NB datasets showed enrichment for Falconi anemia pathway, this is a DNA repair pathway (Tables 1, 2 and 3 ), the functional loss of which is known to result in an increased number of chromosomal breaks. The mismatch repair pathway, responsible for DNA integrity is also known to be dysregulated in cancers. Furthermore, DNA integrity is one of the key functions of the G2/M checkpoint [34] and is lost if cells rapidly or abruptly leave the $\mathrm{S}$ phase. We have shown that DLG2 both negatively correlates to cell cycle gene expression (Fig. 2a) and regulates the cell cycle, namely by slowing the progression of cells through the G2/M checkpoint (Fig. 5c and $\mathrm{f}$ ).

As with the other candidate 11q tumor suppressor genes we have tried to detect a suitable second hit or 


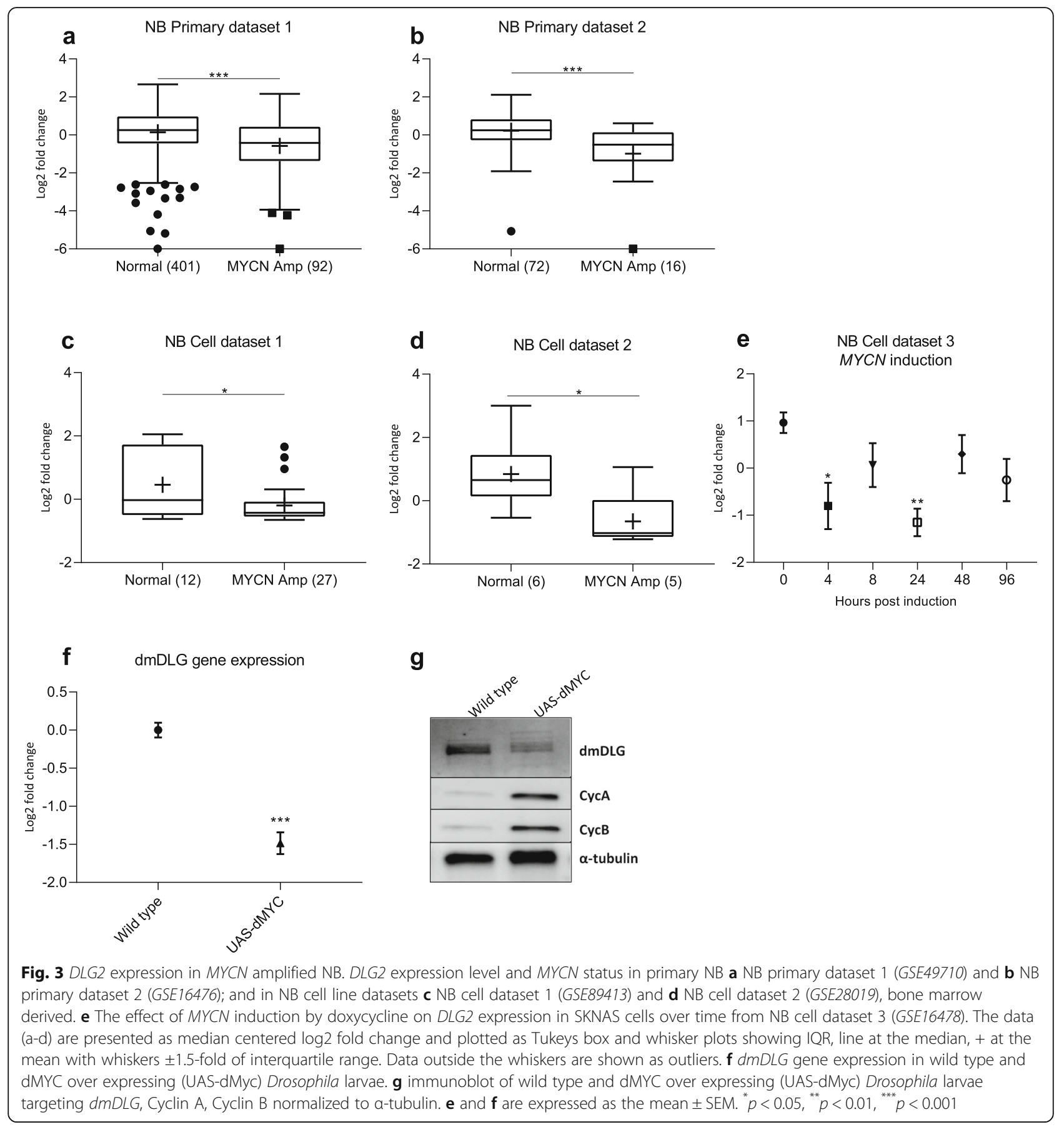

evidence of haploinsufficiency. We have investigated methylation of the CpG island promoter for DLG2 to determine if there was any discernable differential methylation in the NB samples. We were unable to find any difference in the $\mathrm{CpG}$ island, shore or shelf methylation (Fig. 4c and d). We have been able to detect DLG2 expression for most samples, and furthermore, we have not detected any consistent DLG2 mutations, suggesting that there is no second hit for DLG2 but rather a concentration gradient where the lower the expression the worse prognosis. We could show this overall survival and concentration gradient of $D L G 2$ in Fig. 1f. We could also show that there was no advantage but rather a detriment to the cells when DLG2 was further knocked down in the 11q LOH cell line SKNAS (Fig. 5a and b). Previous studies have shown that restoration of DLG2 resulted in an increase in the number of cells paused at the G2-M DNA checkpoint [26], something that we also 


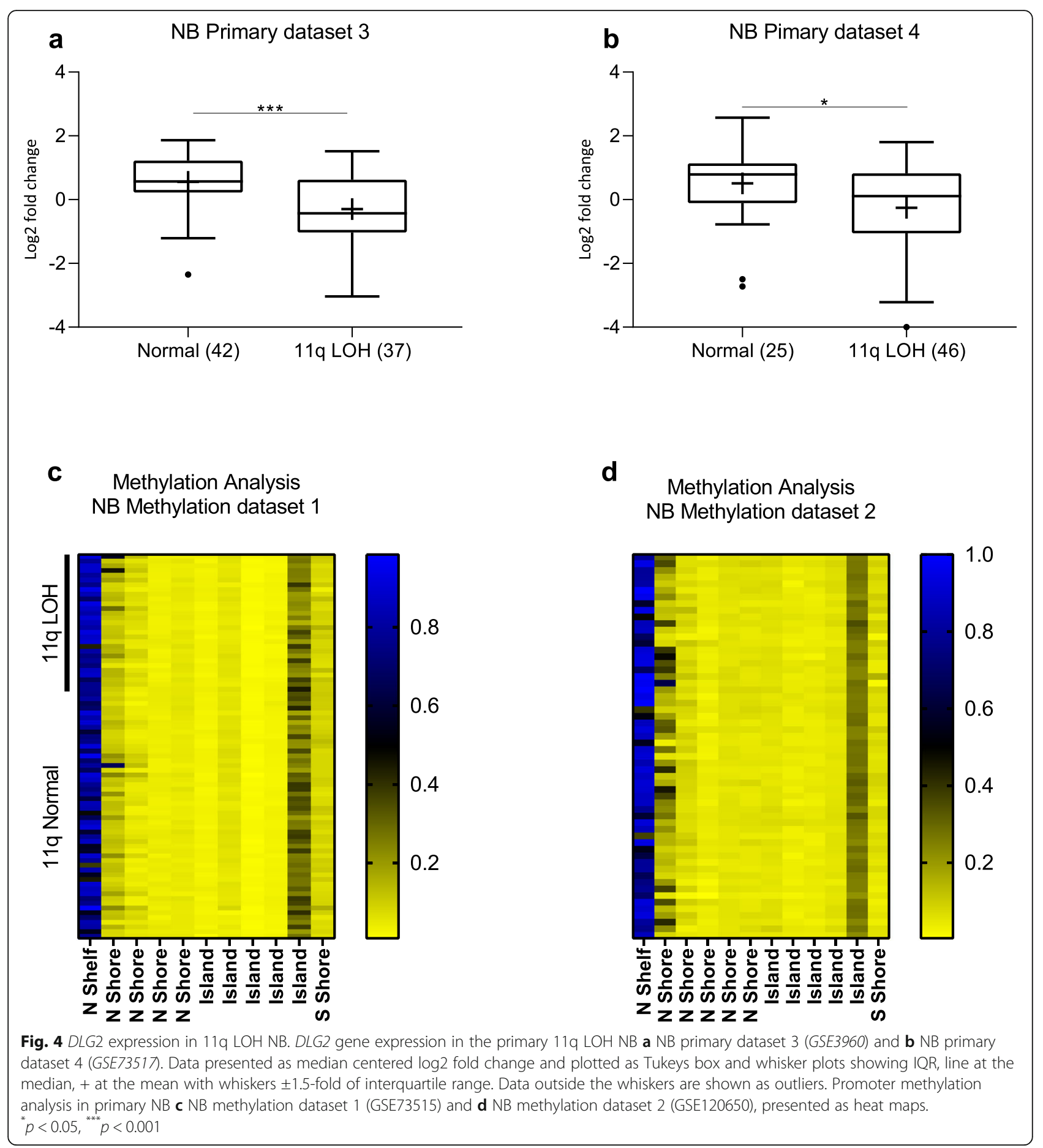

have shown (Fig. 5c and f). Cyclin B protein level exceeding a certain threshold controls passage through this checkpoint. This is consistent with our fly study finding that Cyclin B is highly up regulated with RNAi-dmDLG knockdown (Fig. 5j) and thus allowing DNA damaged cells through the G2-M checkpoint. Furthermore, the GSEA results showed that DLG2 expression level is inversely correlated to cell cycle and DNA replication genes, further strengthening our functional studies. Interestingly, the tumor suppressor candidates on $11 \mathrm{q} 23$ include; ATM, CHEK1 and H2AFX, all of which coalesce within the same DNA damage response pathways, which have been shown to be active at the G1 restriction checkpoint. The small amplification of 11q13 has been 

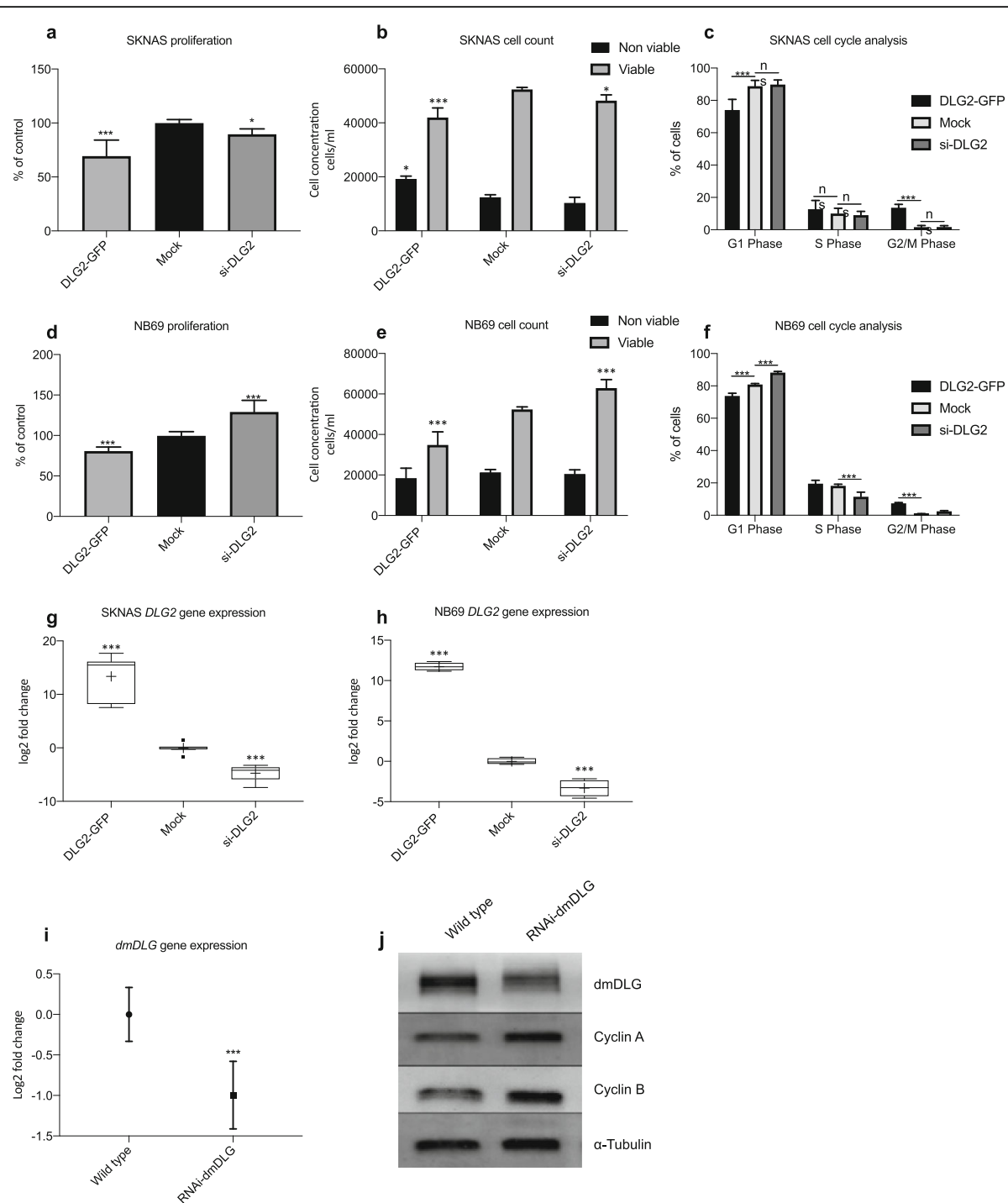

Fig. 5 Cell responses after DLG2 over expression or silencing. Cell responses $48 \mathrm{~h}$ post DLG2 over expression (DLG2-GFP) or silencing (si-DLG2) in SKNAS cells (11q-deleted): a proliferation; $\mathbf{b}$ viable and non-viable cell fraction; c cell cycle analysis. Cell responses $48 \mathrm{~h}$ post DLG2 over expression (DLG2-GFP) or silencing (si-DLG2) in NB69 cells (11q-normal): $\mathbf{d}$ proliferation; e viable and non-viable cell fraction; $\mathbf{f}$ cell cycle analysis. DLG2 gene expression analysis $48 \mathrm{~h}$ post DLG2 over expression (DLG2-GFP) or silencing (si-DLG2) in $\mathbf{g}$ SKNAS cells; $\mathbf{h}$ NB69 cells. i dmDLG gene expression in wild type and dmDLG silenced (RNAi-dmDLG) Drosophila larvae. $\mathbf{j}$ immunoblot of wild type and dmDLG silenced (RNAi-dmDLG) Drosophila larvae targeting dmDLG, Cyclin A, Cyclin B, normalized to a-tubulin. The data in $\mathbf{a}$-f shows the mean and SD. $\mathbf{a}$ and $\mathbf{d}$ are normalized as a percentage of the mock transfection, $n=9$. The data shown is the pooled average of 3 experiments. $\mathbf{g}$ and $\mathbf{h}$ are expressed as median centered log 2 fold change and plotted as Tukeys box and whisker plots showing IQR, line at the median, + at the mean with whiskers \pm 1.5 -fold of interquartile range. ${ }^{*} p<0.05,{ }^{* * *} p<0.001, n=$ not significant

shown to include Cyclin D, a driving factor pushing the cell into the cell cycle [17]. Therefore, the combination of all of the SROs on 11q can regulate the cell cycle at the different checkpoints and therefore the sum of the alterations may sufficiently dysregulate the cell cycle and account for the haploinsufficiency of all of the candidate genes. These findings are in agreement with the reviewed data by Mlakar et al., for 11q deleted NB as they also reached the conclusion that the $11 \mathrm{q}$ deletion is likely going to have haploinsufficiency [11].

\section{Conclusions}

In conclusion, we have provided evidence that gene expression of $D L G 2$ is disrupted in NB, in particular in the aggressive subsets of tumors. It provides a link between $M Y C N$ amplified and 11q-deleted NB, as high levels of MYCN down regulates $D L G 2$ expression, as will loss of one copy of the gene in the 11q-deleted tumors. DLG2 fulfills the criteria of molecular and clinical characteristics to be an 11q tumor suppressor gene functioning as a G2/M checkpoint. 


\section{Supplementary information}

Supplementary information accompanies this paper at https://doi.org/10. 1186/s12964-020-00553-6.

\section{Additional file 1.}

\section{Abbreviations}

DLG: Discs large homolog; LOH: Loss of heterozygosity; MYCN: N-myc protooncogene protein; NB: Neuroblastoma; SRO: Smallest region of overlap; TSG: Tumor suppressor gene

\section{Acknowledgements}

We thank the Swedish Childhood Cancer Fund, Jane and Dan Olsson foundation and Assar Gabrielssons Fund for financial support.

\section{Authors' contributions}

KE generated conception and designed this study and provided technical and material support. AL provided technical support and assistance in study design. SK developed the methodology, performed the assays, analyzed and interpreted the data. SA performed the fly crosses and qPCR. SK and KE organized the data and wrote the manuscript. All authors revised, read and approved the final manuscript.

\section{Funding}

This work was supported by grants from the Swedish Childhood Cancer Fund, Jane and Dan Olsson Foundation and Assar Gabrielsson Fund. The funders were not involved in study design, data collection and analysis, decision to publish, or preparation of the manuscript. Open access funding provided by University of Skövde.

\section{Availability of data and materials}

The datasets analyzed during the current study are available in the 'R2: Genomics Analysis and Visualization Platform repository, [http://r2.amc.nl]

\section{Ethics approval and consent to participate}

Only primary tumor data from publically available sources were used in this study.

\section{Consent for publication}

All authors give consent for the publication of the manuscript.

\section{Competing interests}

The authors declare that they have no competing interests.

\section{Author details}

${ }^{1}$ Translational Medicine, School of Health Sciences, University of Skövde, PO Box 408, SE-54128 Skövde, Sweden. ${ }^{2}$ Translational Bioinformatics, School of Biosciences, University of Skövde, Skövde, Sweden.

\section{Received: 9 January 2020 Accepted: 17 March 2020}

Published online: 20 April 2020

\section{References}

1. Pugh TJ. The genetic landscape of high-risk neuroblastoma. Nat Genet. 2013;3:279.

2. Caren $\mathrm{H}$, Kryh H, Nethander M, Sjoberg R-M, Trager C, Nilsson S, et al. Highrisk neuroblastoma tumors with 11q-deletion display a poor prognostic, chromosome instability phenotype with later onset. Proc National Acad Sciences United States. 2010;9:4323.

3. Parmar R, Wadia F, Yassa R, Zenios M. Neuroblastoma: a rare cause of a limping child. How to avoid a delayed diagnosis? J Pediatr Orthop. 2013; 33(4):e45-51.

4. Smith El, Haase GM, Seeger RC, Brodeur GM. A surgical perspective on the current staging in neuroblastoma--the international neuroblastoma staging system proposal. J Pediatr Surg. 1989:24(4):386-90.

5. Shuangshoti S, Shuangshoti S, Nuchprayoon I, Kanjanapongkul S, Marrano P, Irwin MS, et al. Natural course of low risk neuroblastoma. Pediatr Blood Cancer. 2012;58(5):690-4.
6. Hill-Kayser CE, Tochner Z, Li Y, Kurtz G, Lustig RA, James P, et al. Outcomes after proton therapy for treatment of pediatric high-risk neuroblastoma. Int J Radiat Oncol Biol Phys. 2019;104(2):401-8.

7. Salloum R, Garrison A, von Allmen D, Sheridan R, Towbin AJ, Adams D, et al. Relapsed perinatal neuroblastoma after expectant observation. Pediatr Blood Cancer. 2015:62(1):160-2.

8. Hungate EA, Applebaum MA, Skol AD, Vaksman Z, Diamond M, McDaniel L, et al. Evaluation of Genetic Predisposition for MYCN-Amplified Neuroblastoma. J Natl Cancer Inst. 2017:109:10.

9. Chang X, Zhao Y, Hou C, Glessner J, McDaniel L, Diamond MA, et al. Common variants in MMP20 at 11q22.2 predispose to 11q deletion and neuroblastoma risk. Nat Commun. 2017;8(1):569

10. Maris JM, Guo C, White PS, Hogarty MD, Thompson PM, Stram DO, et al. Allelic deletion at chromosome bands 11 q14-23 is common in neuroblastoma. Med Pediatr Oncol. 2001:36(1):24-7.

11. Mlakar V, Jurkovic Mlakar S, Lopez G, Maris JM, Ansari M, Gumy-Pause F. 11q deletion in neuroblastoma: a review of biological and clinical implications. Mol Cancer. 2017;16(1):114

12. Knudson AG Jr. Mutation and cancer: statistical study of retinoblastoma. Proc Natl Acad Sci U S A. 1971;68(4):820-3.

13. Rushlow DE, Mol BM, Kennett JY, Yee S, Pajovic S, Theriault BL, et al. Characterisation of retinoblastomas without RB1 mutations: genomic, gene expression, and clinical studies. Lancet Oncol. 2013;14(4):327-34.

14. Ando K, Ohira M, Ozaki T, Nakagawa A, Akazawa K, Suenaga Y, et al. Expression of TSLC1, a candidate tumor suppressor gene mapped to chromosome 11q23, is downregulated in unfavorable neuroblastoma without promoter hypermethylation. Int J Cancer. 2008;123(9):2087-94.

15. Mandriota SJ, Valentijn LJ, Lesne L, Betts DR, Marino D, Boudal-Khoshbeen $M$, et al. Ataxia-telangiectasia mutated (ATM) silencing promotes neuroblastoma progression through a MYCN independent mechanism. Oncotarget. 2015;6(21):18558-76.

16. Guo C, White PS, Weiss MJ, Hogarty MD, Thompson PM, Stram DO, et al. Allelic deletion at 11q23 is common in MYCN single copy neuroblastomas. Oncogene. 1999:18(35):4948.

17. Javanmardi N. Genomic instability and genetic heterogeneity in neuroblastoma. Doctoral thesis: University of Gothenburg Sahlgrenska Academy; 2017. http://hdl.handle.net/2077/52856.

18. Roberts S, Delury C, Marsh E. The PDZ protein discs-large ( DLG): the ' Jekyll and Hyde' of the epithelial polarity proteins. FEBS J. 2012;279(19):3549-58.

19. Ludford-Menting MJ, Thomas SJ, Crimeen B, Harris LJ, Loveland BE, Bills M, et al. A functional interaction between CD46 and DLG4: a role for DLG4 in epithelial polarization. J Biol Chem. 2002;277(6):4477-84.

20. Liu J, Li J, Ren Y, Liu P. DLG5 in cell polarity maintenance and cancer development. Int J Biol Sci. 2014;10(5):543-9.

21. Papagiannouli F, Mechler BM. Refining the role of Lgl, Dlg and Scrib in Tumor Suppression and Beyond: Learning from the Old Time Classics. InTech; 2012.

22. Woods DF, Hough C, Peel D, Callaini G, Bryant PJ. Dlg protein is required for junction structure, cell polarity, and proliferation control in Drosophila epithelia. J Cell Biol. 1996;134(6):1469-82.

23. Humbert $P$, Russell $\mathrm{S}$, Richardson $\mathrm{H}$. Dlg, scribble and Lgl in cell polarity, cell proliferation and cancer. Bioessays. 2003;25(6):542-53.

24. Lebedeva LI, Dubatolova TD, Omel'ianchuk LV. Drosophila melanogaster imaginal discs mitotic anomalies induced by tumor-supressor dlg silencing construction. Tsitologiia. 2013:55(6):406-13.

25. Albertson R, Doe CQ. Dlg, Scrib and Lgl regulate neuroblast cell size and mitotic spindle asymmetry. Nat Cell Biol. 2003;5(2):166-70.

26. Shao YW, Wood GA, Lu J, Tang QL, Liu J, Molyneux S, et al. Cross-species genomics identifies DLG2 as a tumor suppressor in osteosarcoma. Oncogene. 2019;38(2):291-8.

27. Zhuang RJ, Bai XX, Liu W. MicroRNA-23a depletion promotes apoptosis of ovarian cancer stem cell and inhibits cell migration by targeting DLG2. Cancer Biol Ther. 2019:1-15.

28. Turnbull C, Perdeaux ER, Pernet D, Naranjo A, Renwick A, Seal S, et al. A genome-wide association study identifies susceptibility loci for Wilms tumor. Nat Genet. 2012:44(6):681-4

29. Ongena K, Das C, Smith JL, Gil S, Johnston G. Determining cell number during cell culture using the scepter cell counter. JoVE. 2010;45:2204.

30. Orian A, Delrow JJ, Rosales Nieves AE, Abed M, Metzger D, Paroush Z, et al. A Myc-Groucho complex integrates EGF and notch signaling to regulate neural development. Proc Natl Acad Sci U S A. 2007;104(40):15771-6. 
31. Valentijn LJ, Koster J, Haneveld F, Aissa RA, van Sluis P, Broekmans ME, et al. Functional MYCN signature predicts outcome of neuroblastoma irrespective of MYCN amplification. Proc Natl Acad Sci U S A. 2012;109(47):19190-5.

32. Murphy DM, Buckley PG, Bryan K, Das S, Alcock L, Foley NH, et al. Global

MYCN transcription factor binding analysis in neuroblastoma reveals association with distinct E-box motifs and regions of DNA hypermethylation. PLoS One. 2009;4(12):e8154.

33. Planells-Ferrer L, Urresti J, Soriano A, Reix S, Murphy DM, Ferreres JC, et al. MYCN repression of lifeguard/FAIM2 enhances neuroblastoma aggressiveness. Cell Death Dis. 2014;5:e1401.

34. Hou SQ, Ouyang M, Brandmaier A, Hao H, Shen WH. PTEN in the maintenance of genome integrity: From DNA replication to chromosome segregation. Bioessays. 2017;39:10.

\section{Publisher's Note}

Springer Nature remains neutral with regard to jurisdictional claims in published maps and institutional affiliations.

Ready to submit your research? Choose BMC and benefit from:

- fast, convenient online submission

- thorough peer review by experienced researchers in your field

- rapid publication on acceptance

- support for research data, including large and complex data types

- gold Open Access which fosters wider collaboration and increased citations

- maximum visibility for your research: over $100 \mathrm{M}$ website views per year

At BMC, research is always in progress.

Learn more biomedcentral.com/submissions 